\title{
Differential phenotyping of Brucella species using a newly developed semi-automated metabolic system
}

Sascha Al Dahouk ${ }^{1,2^{*}}$, Holger C Scholz ${ }^{3}$, Herbert Tomaso ${ }^{4}$, Peter Bahn ${ }^{1}$, Cornelia Göllner ${ }^{1}$, Wolfram Karges ${ }^{2}$, Bernd Appel ${ }^{1}$, Andreas Hensel ${ }^{1}$, Heinrich Neubauer ${ }^{4}$, Karsten Nöckler ${ }^{1}$

\begin{abstract}
Background: A commercial biotyping system (Taxa Profile ${ }^{\mathrm{TM}}$, Merlin Diagnostika) testing the metabolization of various substrates by bacteria was used to determine if a set of phenotypic features will allow the identification of members of the genus Brucella and their differentiation into species and biovars.

Results: A total of 191 different amines, amides, amino acids, other organic acids and heterocyclic and aromatic substrates (Taxa Profile ${ }^{\mathrm{TM}}$ A), 191 different mono-, di-, tri- and polysaccharides and sugar derivates (Taxa Profile ${ }^{\mathrm{TM}} \mathrm{C}$ ) and 95 amino peptidase- and protease-reactions, 76 glycosidase-, phosphatase- and other esterase-reactions, and 17 classic reactions (Taxa Profile ${ }^{\mathrm{TM}} \mathrm{E}$ ) were tested with the 23 reference strains representing the currently known species and biovars of Brucella and a collection of 60 field isolates. Based on specific and stable reactions a 96-well "Brucella identification and typing" plate (Micronaut ${ }^{\mathrm{TM}}$ ) was designed and re-tested in 113 Brucella isolates and a couple of closely related bacteria.

Brucella species and biovars revealed characteristic metabolic profiles and each strain showed an individual pattern. Due to their typical metabolic profiles a differentiation of Brucella isolates to the species level could be achieved. The separation of $B$. canis from B. suis bv 3, however, failed. At the biovar level, B. abortus bv 4, 5, 7 and B. suis bv 1-5 could be discriminated with a specificity of $100 \%$. B. melitensis isolates clustered in a very homogenous group and could not be resolved according to their assigned biovars.

Conclusions: The comprehensive testing of metabolic activity allows cluster analysis within the genus Brucella. The biotyping system developed for the identification of Brucella and differentiation of its species and biovars may replace or at least complement time-consuming tube testing especially in case of atypical strains. An easy to handle identification software facilitates the applicability of the Micronaut ${ }^{\mathrm{TM}}$ system for microbiology laboratories.
\end{abstract}

\section{Background}

Brucella spp. are the causative agents of brucellosis, one of the major bacterial zoonotic diseases that is responsible for reproductive failure in animals leading to tremendous economic losses and for a potentially debilitating infection in man. Furthermore, Brucella is listed as category B bioterrorism agent.

Species and biovar classification of brucellae is historically based on natural host preference and phenotypic traits, i.e. $\mathrm{CO}_{2}$ requirement, $\mathrm{H}_{2} \mathrm{~S}$ production, urease

\footnotetext{
* Correspondence: Sascha.Al-Dahouk@gmx.de

'Federal Institute for Risk Assessment, Diedersdorfer Weg 1, D-12277 Berlin, Germany

Full list of author information is available at the end of the article
}

activity, dye-sensitivity, lysis by Brucella-specific bacteriophages, agglutination with monospecific antisera, and oxidative metabolic patterns [1-3]. In concordance with this biotyping scheme the genus Brucella (B.) currently comprises the six classical species $B$. melitensis bv 1-3 (predominantly isolated from sheep and goats), B. abortus bv 1-7 and 9 (from cattle and other Bovidae), B. suis bv 1-3 (from pigs), bv 4 (from reindeer) and bv 5 (from small ruminants), B. canis (from dogs), B. ovis (from sheep), and B. neotomae (from desert wood rats) [4]. Further, two novel species of marine origin, $B$. pinnipedialis (from seals) and $B$. ceti (from dolphins and whales) [5], and B. microti at first isolated from the common vole Microtus arvalis [6], then from red foxes

\section{(Ciomed Central}


(Vulpes vulpes) [7] and also directly from soil [8] have been added to the genus. Most recently $B$. inopinata sp. nov. isolated from a breast implant wound of a female patient has been described as a new species with so far unknown animal reservoir [9].

A biotyping assay useful for Brucella identification and species differentiation must consequently be able to identify the rising number of upcoming new species as well as single atypical strains which do not fit within the pre-existing scheme $[10,11]$. In addition, clinically relevant and closely related bacteria of other genera should be discriminated. Using commercially available rapid bacterial identification systems such as the API $20 \mathrm{NE}^{\bullet}$ (BioMerieux, Nürtingen, Germany) which include a restricted number of biochemical tests Brucella spp. may be misidentified e.g. as Psychrobacter phenylpyruvicus (formerly Moraxella phenylpyruvica) [12] or Ochrobactrum anthropi [13].

The aim of our study was to develop a miniaturised semi-automated system for the reliable identification of members of the genus Brucella and the differentiation of its species based on comprehensive metabolic activity testing.

\section{Results}

The Taxa Profile ${ }^{\mathrm{Tm}}$ system testing the utilization of amino acids (A plates) and carbohydrates (C plates) as well as other enzymatic reactions (E plates) [Additional files 1, 2 and 3] revealed a very high biodiversity among the closely related species and biovars of the genus Brucella (Figure 1A, [Additional files 4, 5 and 6] ). The stability of metabolic profiles significantly varied between the different species and biovars, yet most of the stable markers were found in the Taxa Profile ${ }^{\mathrm{mx}} \mathrm{E}$ plate. Differences between cultures of the same strain were most frequently observed in the species $B$. abortus and $B$. microti, and in biovar 1 of B. suis. A total of 196 out of 570 biochemical reactions proved to be both stable and discriminatory, and showed differences in the metabolism of the 23 Brucella reference strains or helped to distinguish Brucella spp. from closely related bacteria such as Ochrobactrum spp. In general, the broadest metabolic activity could be observed for strains of the species $B$. suis, $B$. microti, and $B$. inopinata. In contrast, the metabolic activity of $B$. ovis, B. neotomae and B. pinnipedialis was low.

The comprehensive biotyping of the reference strains resulted in clusters agreeing in principle with the present conception of the genus Brucella (Figure 1A). A subset of 93 substances which preserved the clustering of the reference strains and achieved a satisfying discrimination was consecutively selected (Figures 2 and 1B). The newly configured 96 -well plate assay tested for 29 aminopeptidases, 2 phosphatases, 4 glucosidases,
1 esterase, and the metabolism of 11 monosaccharides, 3 disaccharides, 7 sugar derivates, 15 amino acids, 11 organic acids, 1 salt, 1 amino acid derivate, 1 peptide, and 1 base. In addition, 6 classical reactions, i.e. nitrite, nitrate, pyrazinamidase, Voges-Proskauer medium, urease and $\mathrm{H}_{2} \mathrm{~S}$ production, and three controls, i.e. peptidase control, pyrazinamidase control and assimilation control were included.

Glu(pNA)-OH (ENAOH), Pyr-pNA (PYRNA) (constantly negative reaction), and $\mathrm{H}$-hydroxyprolin- $\beta \mathrm{NA}$ (HP) (constantly strong positive reaction) turned out to be key substances useful for the identification of the genus Brucella and its differentiation from other bacteria [Additional file 7].

A stable negative reaction for D-threitol (D-TOL) and mostly positive reactions for L-alanine (L-Ala), D-alanine (D-Ala), propionic acid (Propn), L-proline (L-Pro), D-proline (D-Pro), and D-serine (D-Ser) could be observed in B. melitensis. B. microti which also makes use of alanine and proline could be separated from $B$. melitensis by a constantly negative reactivity for Propn and D-Ser. A positive myo-inositol (INOL) reaction seemed to be characteristic for most $B$. melitensis strains and B. inopinata. Bis-p-nitrophenyl phosphate $\mathrm{pH} 7.5$ (BISPH7), p-nitrophenyl phosphate di(2-amino-2-ethyl1,3-propanediol) pH 7.5 (PHOS7), and p-nitrophenyl-ad-glucopyranoside $\mathrm{pH} 7.5$ (aGLU7) were found positive frequently in $B$. suis and regularly in B. microti strains, variable in $B$. melitensis and mostly negative in $B$. abortus. Glutarate (Gluta) and mesaconic acid (Mesac) which were almost exclusively metabolized by $B$. microti might be helpful for further differentiation. P-nitrophenyl-a-d-glucopyranoside pH 5.5 (aGLU5) and p-nitrophenyl-n-acetyl- $\beta$-d-glucosaminide $\mathrm{pH} 7.5$ (CHIT7) showed weak positive reactions in $B$. suis and $B$. canis and strong positive reactions in B. microti and B. inopinata. B. microti and B. inopinata exhibited outstanding metabolic capabilities in comparison to all other brucellae, sharing a series of reactions with $O$. anthropi and $O$. intermedium. Most remarkably, both species were strongly positive in the Voges-Proskauer reaction. The slow growing strains of the B. ovis group did not metabolize any carbohydrates except for D-glucose-Lcysteine (GLUCY), L(+)-arabinose (L-ARA), D-TOL, and adonite (ADON) and only a few amino acids. In addition, $B$. ovis strains were usually not able to deoxidize nitrite (NTI, nitrite reduction) and nitrate (NTA, nitrate reduction). Ac-Gly-Lys- $\beta$ NA (AcGK) tested strongly positive in $B$. ovis and $B$. canis whereas Trp$\beta N A(W)$ regularly tested negative in these species as compared to all other Brucella spp. In comparison with other species $B$. pinnipedialis was weak in metabolizing $\mathrm{D}(-)$-ribose (D-RIB), D(-)-arabinose (D-ARA), D (+)-glucose (D-GLU), L-ARA, D(+)-galactose (D-GAL), 


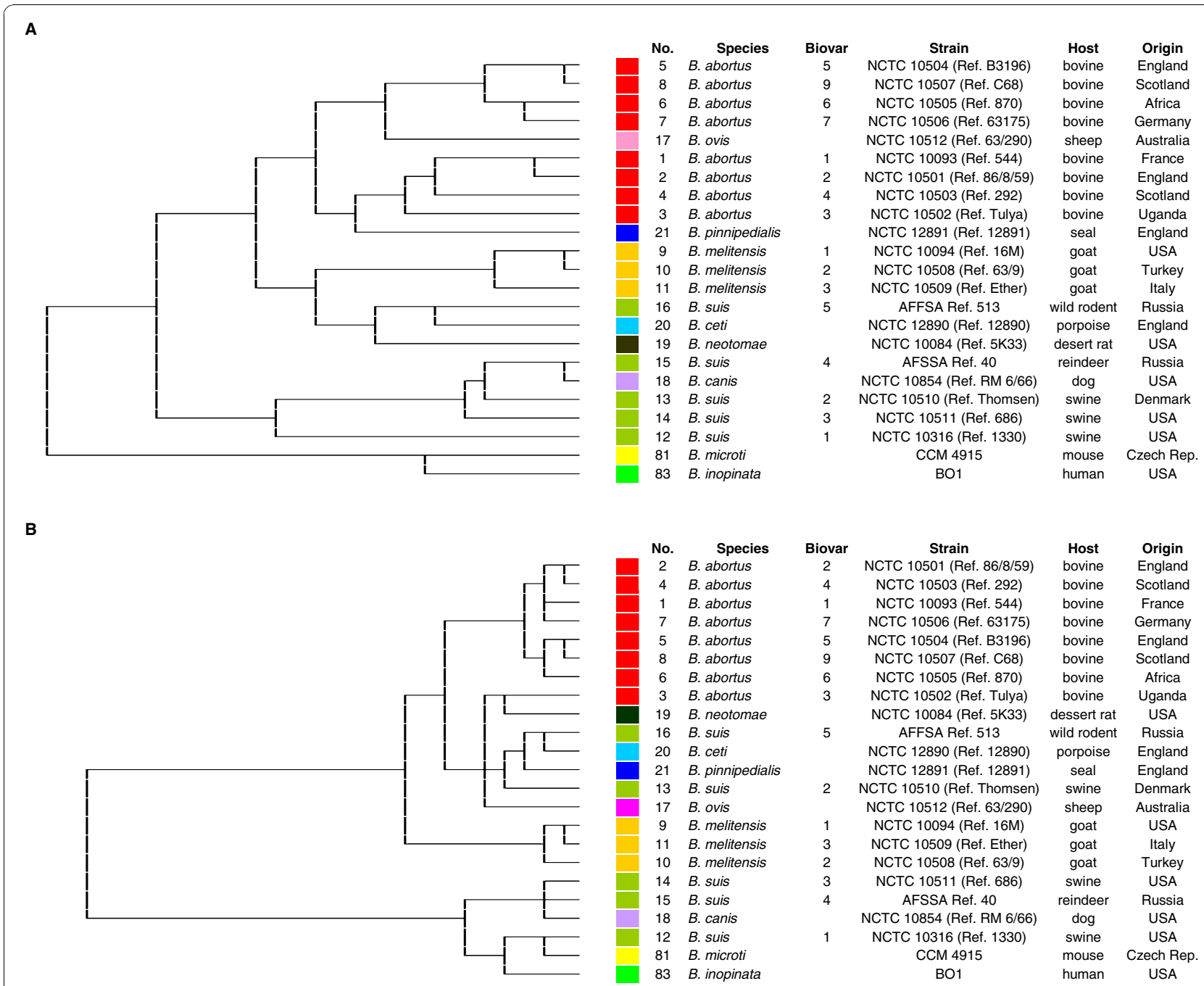

Figure 1 Cluster analysis of Brucella reference strains based on biochemical reactions. Cluster analysis of the 23 Brucella reference strains based on 570 (A) and 93 (B) biochemical reactions tested with the Taxa Profile ${ }^{\mathrm{TM}}$ system (plate A, C, and E) and the newly developed Brucella specific Micronaut ${ }^{T M}$ microtiter plate, respectively. Hierarchical cluster analysis was performed by the Ward's linkage algorithm using the binary coded data based on the empirically set cut-off.

$\mathrm{D}(+)$-xylose (D-XYL), and a-D-talose (D-TAL). B. ceti and $B$. pinnipedialis showed significantly different carbohydrate utilization patterns. $B$. neotomae was the only species tested negative for d-Ala-pNA (DANA), GlypNA (GNA), Leu-pNA (LNA), Lys-pNA (KNA), Lys$\beta N A(K)$, and Gly-Gly- $\beta$ NA (GG). Like B. neotomae the two yet unidentified strains isolated from foxes were negative for DANA and GNA. Despite of genetic consistency with the genus Brucella (data not shown) these two strains completely differed in their metabolic profile from the species described to date.

The panel of 93 discriminating reactions was re-evaluated for its usefulness in the identification of Brucella and the differentiation of its species and biovars using a broad spectrum of well characterized field strains. Both inter- and intra-assay variability were ascertained to be negligible. Results of the cluster analysis of the 113 strains investigated regarding their ability to metabolize the 93 selected substances supported our findings in the smaller collection of Brucella reference strains (Figure 3). Based on the metabolic profiles determined by the Brucella specific 96-well Micronaut $^{\mathrm{TM}}$ plate, B. melitensis and B. abortus isolates fell into two distinct groups (Figure 3). B. suis (except for biovar 5) could be found in another group but the biovars 1 , and 3 and 4 gathered together with $B$. inopinata and $B$. canis isolates, respectively. $B$. suis bv 2 could be separated by its substrate assimilation pattern. $B$. suis bv 5 showed metabolic traits similar to B. ovis, B. neotomae and the marine mammal strains. Each Brucella strain investigated revealed an individual metabolic profile. 


\begin{tabular}{|c|c|c|c|c|c|c|c|c|c|c|c|c|}
\hline & 1 & 2 & 3 & 4 & 5 & 6 & 7 & 8 & 9 & 10 & 11 & 12 \\
\hline A & DANA & HP & $\mathrm{N}$ & D & aGLU7 & D-RIB & D-TOL & INOL & GuaSu & AcArg & D-Pro & NTI \\
\hline B & GNA & $\mathrm{IL}$ & w & $E$ & aMAL7 & D-ARA & ADON & INON & L-Cyss & Glyx & Fuma & NTA \\
\hline c & LNA & $\mathrm{K}$ & EH & Pyr & aGLU5 & D-GLU & SUCR & Galli & D-Ala & L-Ser & D-Ser & PCA \\
\hline D & KNA & $\mathrm{RR}$ & AcKA & $\beta A$ & aXYL7 & L-ARA & MALTO & diLac & Propn & HipArg & Glyc & VP \\
\hline $\mathbf{E}$ & GPNA & GG & dAdA & $\mathrm{V} 4 \mathrm{M}$ & CHIT7 & DOGAL & D-FRU & Acet & L-Ala & L-Caro & Adeni & Urease \\
\hline $\mathbf{F}$ & AFPNA & $P$ & v & PepK & EROL & D-GAL & DNSOL & L-Asn & $\beta$ HBut & Gly & Gluta & $\mathrm{H}_{2} \mathrm{~S}$ \\
\hline G & ENAOH & AcGK & 1 & BISPH7 & L-FUC & $D-X Y L$ & LYXA & L-Glu & D-Asn & Adipa & Mesac & PCC \\
\hline H & PYRNA & AFPA & VTS & PHOS7 & GLUCY & D-TAL & PAL & AlaGin & L-Arg & L-Pro & D-His & $\mathrm{ACO}$ \\
\hline \multicolumn{4}{|c|}{ carbohydrates } & amino acid & & & \multicolumn{3}{|c|}{ other enzymatic reactions } & & controls & \\
\hline & & \multicolumn{2}{|c|}{ monosaccharides } & & amino aci & & & \multicolumn{3}{|c|}{ aminopeptidases with pNA } & & \\
\hline & & \multicolumn{2}{|c|}{ disaccharides } & & organic ac & & & \multicolumn{3}{|c|}{ aminopeptidases with $\beta N A$} & & \\
\hline & & \multicolumn{2}{|c|}{ sugar derivates } & & salts & & & \multicolumn{3}{|c|}{ glucosidases } & & \\
\hline & & & & & \multicolumn{2}{|c|}{ amino acid derivates } & & \multicolumn{3}{|c|}{ phosphatases } & & \\
\hline & & & & & \multicolumn{2}{|l|}{ peptides } & & \multicolumn{3}{|l|}{ esterases } & & \\
\hline & & & & & \multicolumn{2}{|c|}{ basic substrates } & & \multicolumn{3}{|c|}{ classical reactions } & & \\
\hline
\end{tabular}

Figure 2 The Brucella specific Micronaut ${ }^{\mathrm{TM}}$ microtiter plate. Design of the newly developed Brucella specific Micronaut ${ }^{\mathrm{TM}}$ microtiter plate including 93 selected substances.

Using the newly developed Brucella specific Micronaut $^{\text {tix }}$ biotyping assay, $B$. abortus bv 4,5 , and $7, B$. suis bv 1-5, B. ovis, B. neotomae, B. pinnipedialis, B. ceti, $B$. microti, and $B$. inopinata could be discriminated within the genus with a specificity of $100 \%$ (Table 1 ). In contrast, members of the three B. melitensis biovars formed a homogenous group. Although the metabolic activity of B. melitensis strains did not correlate with the classical biotyping scheme, subgroups within the species could still be defined (Figure 3). Gram-negative microorganisms other than brucellae e.g. Ochrobactrum intermedium, O. anthropi, Yersinia enterocolitica O:9, and Acinetobacter lwoffii showed differing oxidative metabolic profiles and could clearly be distinguished from Brucella spp. in our experimental setting. Furthermore, a screening of the Micronaut-IDS database (Merlin Diagnostika) which is a widely used rapid identification system for Gram-negative and Gram-positive bacteria clearly discriminated brucellae from other bacterial taxa on the basis of four enzymatic reactions i.e. HP, Pyr$\beta$ NA (Pyr), urease, and NTA [Additional file 8, only clinically relevant bacteria are shown].

The biotyping results were independent of the host and the geographic origin of Brucella isolates.

\section{Discussion}

\section{Classical phenotyping and metabolic markers of Brucella} spp

Although Brucella is a monophyletic genus, apparent differences between its species do exist e.g. host specificity and pathogenicity. Nowadays, Brucella species and biovars are distinguished by a limited number of microbiological tests measuring quantitative or qualitative differences of dye bacteriostasis, hydrogen sulfide production, urea hydrolysis, carbon dioxide requirement, bacteriophage sensitivity and agglutinin absorption. For at least half a century these microbiological procedures have not changed, although various new Brucella species showing variable phenotypic traits have been detected and new diagnostic methods have been developed.

Neither the classical biochemical tests nor antigenic properties and phage-sensitivity can be considered a reliable guide to the identification of Brucella species. Contradictory results were often reported [14]. However, variations in $\mathrm{H}_{2} \mathrm{~S}$ production, $\mathrm{CO}_{2}$ requirement, a change in dye tolerance or atypical surface antigens i.e. inconsistent $\mathrm{A}$ and $\mathrm{M}$ antigens usually do not affect the oxidative metabolic pattern of a strain $[15,16]$. Metabolic activities have proven to be stable parameters allowing unambiguous species identification, particularly in strains which show conflicting identities by conventional determinative methods [14,17-19]. In addition, differing metabolism may help to describe new species $[6,9,20]$. In our series, two strains isolated from foxes in Austria (strain no. 110 and 111) which displayed an atypical metabolic pattern could be identified.

Oxidative metabolic profiles remain qualitatively stable for long periods of time and usually show no change in characteristic patterns after in vivo and in vitro passages [21]. However, quantitative differences in the oxidative metabolic rate of monosaccharides have been observed after multiple passages in vitro [21]. Variants in the oxidative metabolic pattern found among different CFUs of the same strain have been described in varying frequencies depending on Brucella species and biovars [22]. In our experiments, B. suis bv 1 showed the highest intrastrain variability in its enzymatic activity (data not shown).

Despite the stability of the metabolic markers and their consecutive usefulness in diagnostic assays, studies describing the differences in the metabolism of Brucella spp. have not been conducted for decades as the 


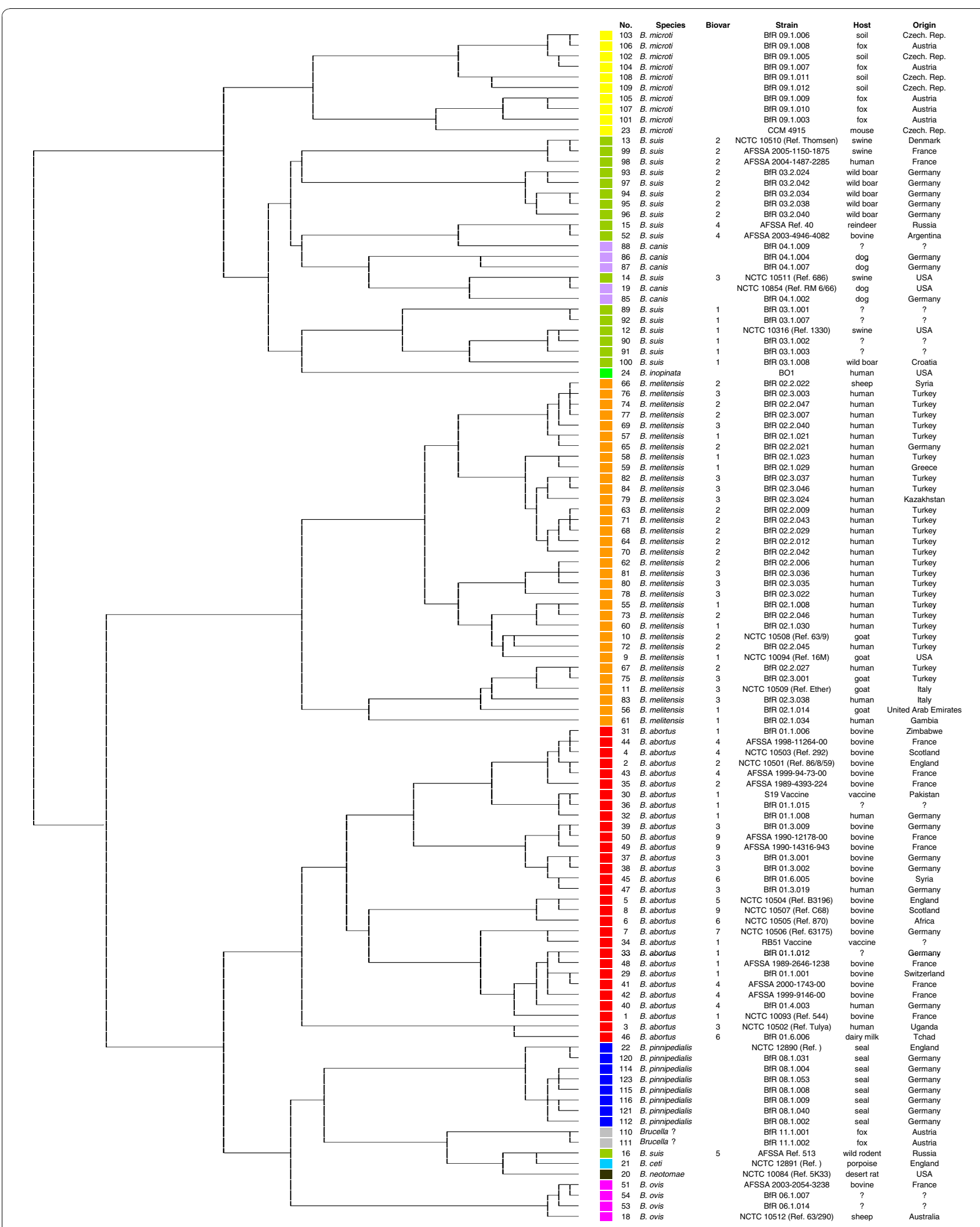

Figure 3 Cluster analysis of Brucella field isolates based on biochemical reactions. Cluster analysis of 113 Brucella strains including the reference strains and two isolates of a potentially new species that originated from Austrian foxes based on 93 biochemical reactions tested with the newly developed Brucella specific Micronaut ${ }^{\mathrm{TM}}$ microtiter plate. Hierarchical cluster analysis was performed by the Ward's linkage algorithm using the binary coded data based on the empirically set cut-off. 
Table 1 Specificity of the Brucella specific Micronaut ${ }^{\mathrm{TM}}$ microtiter plate.

\begin{tabular}{|c|c|c|c|c|}
\hline \multicolumn{2}{|c|}{ Brucella spp. } & \multicolumn{3}{|c|}{ Specificity in \% } \\
\hline \multirow[t]{4}{*}{ Species } & Biovars & $\begin{array}{c}\text { Biovar } \\
\text { differentiatio }\end{array}$ & $\begin{array}{r}\mathrm{Sp} \\
\text { differ }\end{array}$ & tion \\
\hline & 1 & $\underline{0}$ & & \\
\hline & 2 & $\underline{75}$ & & \\
\hline & 3 & $\underline{90}$ & & \\
\hline \multirow[t]{6}{*}{ B. abortus } & 4 & 100 & 100 & \\
\hline & 5 & $\underline{100}$ & & \\
\hline & 6 & $\underline{0}$ & & \\
\hline & 7 & $\underline{100}$ & & \\
\hline & 9 & 0 & & \\
\hline & 1 & $\underline{19}$ & 100 & \\
\hline \multirow[t]{4}{*}{ B. melitensis } & 2 & 89 & & \\
\hline & 3 & 64 & & \\
\hline & 1 & $\underline{100}$ & 100 & 99 \\
\hline & 2 & $\underline{100}$ & & \\
\hline \multirow[t]{3}{*}{ B. suis } & 3 & 100 & & \\
\hline & 4 & 100 & & \\
\hline & 5 & 100 & & \\
\hline B. ovis & & & 100 & \\
\hline B. canis & & & $\underline{60}$ & \\
\hline B. neotomae & & & 100 & \\
\hline B. ceti & & & 100 & \\
\hline B. pinnipedialis & & & 100 & \\
\hline B. microti & & & 100 & \\
\hline B. inopinata & & & 100 & \\
\hline
\end{tabular}

Specificity of the Micronaut ${ }^{\mathrm{T} M}$ system to differentiate Brucella species and biovars.

classical laboratory techniques are labour-intensive and very demanding. Especially Warburg manometry which is carried out in a respirometer measuring oxygen uptake has been widely used to determine oxidative metabolic patterns in order to describe and differentiate species, biovars, and atypical strains of the genus Brucella. Formerly, manometric studies on the metabolic activity of brucellae helped to quantitatively define the species classified within the genus [23]. However, due to the demanding techniques applied only a restricted number of strains and reactions were tested and various substrates e.g. D-asparagine, L-proline, adonitol, fructose and glucose were regarded as not useful for species and biovar differentiation $[23,24]$. In the comprehensive setting of this study most of these substrates also proved their usefulness.

Manometric studies have confirmed that a characteristic oxidative pattern for Brucella species exists whereas specific profiles for the biovars have not yet been described except for B. suis bv 1-4 [25]. Using the Micronaut $^{\mathrm{TM}}$ system we were able to discriminate $B$. abortus bv 4,5 , and $7, B$. suis bv $1-5, B$. ovis, $B$. neotomae, B. pinnipedialis, B. ceti, B. microti and B. inopinata with a specificity of $100 \%$. However, differentiation among the $B$. melitensis biovars was impossible as, according to their oxidative metabolic activity, they form a very homogenous group. The results of the cluster analysis based on our biotyping data (Figure 3) are in general concordance with the genotyping data acquired by Multiple Loci VNTR (Variable Number of Tandem Repeats) Analysis (MLVA) [26]. Neither biotyping nor genotyping proved a biovar specific clustering in B. melitensis strains [27]. Although we tested a substantial number of biochemical reactions we may have chosen the wrong set of substrates for the differentiation of B. melitensis strains, but the separation of this species in three biovars could also be somehow artificial.

\section{Biotyping of Brucella spp. using commercially available assays}

If biological traits such as enzymatic activities are tested all potential variables must be reduced to a minimum to avoid intra- and inter-assay variations which may occur in addition to minimal biological variations. Commercial test systems offer a large number of quality controls both in the production chain and under experimental conditions.

Commercially available microtiter plates coated with various substrates to characterize the metabolic pattern of bacteria have already been used to describe new species of the genus Brucella e.g. the Biolog ${ }^{\mathrm{Tm}}$ system for $B$. ceti [28] and the Micronaut ${ }^{\mathrm{TM}}$ system for B. microti and $B$. inopinata [6,9]. However, comprehensive metabolic studies including all currently known species and biovars are rare.

Using the Biolog ${ }^{\text {TM }}$ GN MicroPlate system (Biolog, CA, USA) based on 44 differentially oxidized substrates, $B$. melitensis, B. abortus and B. suis isolates could be grouped into taxons identical with the presently recognized species [29]. However, only a restricted number of strains $(\mathrm{n}=35)$ were tested and biovars were not differentiated. In a larger strain collection $(n=71)$ which included all biovars of the six classical Brucella species only $50 \%$ of the strains were correctly identified confirming the poor specificity of this commercially available, substrate mediated, tretrazolium identification technique [30]. López-Merino and colleagues used the Biotype $100^{\mathrm{mm}}$ carbon substrate assimilation system (bioMérieux, Marcy-L'Etoile, France) which comprises 99 carbohydrates, organic acids and other carbon substrates to discriminate B. melitensis, B. abortus, B. suis and B. canis [31]. Using the most discriminating carbon substrates i.e. D-glucose, D-trehalose, D-ribose, palatinose, L-fucose, L-malate, and DL-lactate more than $80 \%$ of the $B$. melitensis and $B$. abortus strains could be correctly identified. Similar to the Brucella specific 
Micronaut $^{\mathrm{Tw}}$ plate designed in this study B. suis and $B$. canis could not always be discriminated. The limited number of field isolates tested per species may have produced inconclusive results, particularly when only reference strains were available which are well known for atypical phenotypic traits. Future studies on larger strain collections may reveal more unique metabolic profiles suitable for species and biovar differentiation and also helpful to discriminate between $B$. suis bv 3 and $B$. canis. Nevertheless, the overall specificity for the identification of Brucella species using the Micronaut ${ }^{\mathrm{mm}}$ system reached $99 \%$.

\section{Experimental conditions potentially interfering with bacterial metabolism and influencing biotyping results}

Many experimental parameters may influence the metabolic activity of bacteria. For instance, oxidative rates may decrease if Brucella is prepared from 48 hours rather than 24 hours cultures [25] because Brucella is able to adapt to starvation. This effect does not seem to be important in the Micronaut ${ }^{\text {tw }}$ system since turbidity is measured reflecting bacterial growth within a period of 48 hours as an indirect parameter for substrate utilization. Consequently, the bacteria have plenty of time to switch on all necessary metabolic pathways. Hence, the metabolic rate of glutamic acid may differ between $B$. abortus and B. melitensis [32] but after $48 \mathrm{~h}$ the substrate is entirely metabolized by both species. For the same reason $B$. suis has been described as inactive in the metabolism of glutamic acid but our results revealed extensive utilization of this substrate at least for the biovars 3-5 whereas the metabolization was variable in the biovars 1 and 2 .

The growth medium can also have an effect on the utilization of substrates and brucellae may operate with alternate metabolic pathways leading to discrepant stimulatory effects in different assays [30]. Therefore, a minimal medium i.e. buffered sodium chloride peptone (from potatoes) solution was used in Taxa Profile ${ }^{\mathrm{Tt}}$ and Micronaut $^{\mathrm{tm}}$ plates to avoid interference with other potential substrates in the culture medium.

The rates of oxidation of various compounds are also strongly dependent on intact bacterial membranes and $\mathrm{pH}$ values [33,34]. In our experiments, asparagines were easily oxidized by most of the Brucella spp., but aspartic acid was not (exceptions were B. suis bv 4, B. microti, and $B$. inopinata). Furthermore, glutamic acid was oxidized, but intermediates in the pathway, such as $\alpha$-ketoglutarate and succinate (except for $B$. microti and $B$. inopinata) were usually not. Lowering the $\mathrm{pH}$ of a reaction mixture containing intact cells of brucellae markedly increased the oxidation rate of these metabolites e. g. L-aspartate, $\alpha$-ketoglutarate, succinate, fumarate, Lmalate, oxaloacetate, pyruvate and acetate [34].
Differences between Brucella species may occur in the $\mathrm{pH}$ range at which the bacteria are able to utilize some of the substrates and therefore labile metabolic profiles can be observed [35]. Nevertheless, such reactions may be helpful for the differentiation of species and biovars if assay conditions are stable. The effect of extracellular adjustment of the $\mathrm{pH}$ upon intracellular enzymatic reactions can be explained by organic acids permeating the cell more readily when undissociated than when ionized. Hence, a $\mathrm{pH}$ change may overcome the permeability barrier for many substrates especially of the Krebs' cycle. For this reason our results do not easily reflect intracellular substrate utilization. In proteomic studies on intracellular brucellae and bacteria grown under stress conditions comparable to the intracellular niche of Brucella, enzymes of the TCA cycle i.e. the succinyl CoA synthetase and aconitate hydratase were found increased [36,37]. In contrast, intermediates of the TCA cycle such as citrate, isocitrate, $\alpha$-ketoglutarate, succinate, malate, fumarate were not generally metabolized in vitro or showed variable metabolization in the different species such as oxaloacetic acid.

Although modelling of the intracellular niche of brucellae is not a topic of this study the Micronaut ${ }^{\mathrm{mm}}$ system might be helpful to investigate differences in the metabolic activity between the species under various growth conditions. This will allow a much deeper insight in the metabolic changes of intracellular compared to extracellular brucellae and will help to understand survival strategies of the pathogen under starvation, microaerobic and acidic conditions. In this context, a negative correlation between metabolic activity and the relative degree of virulence was observed among $B$. abortus strains [38]. Avirulent mutants of $B$. melitensis, B. abortus and $B$. suis that failed to replicate or survive in macrophages or animal models often had mutations in the carbohydrate metabolism [39]. In our study, B. microti which is not known to be human pathogenic was the metabolically most active species.

Independent of the method used a broad agreement can be observed for the utilization of carbohydrates by Brucella spp. whereas the results of the amino acid metabolism are more variable $[3,16]$. Differences in the oxidation rate of different isomers of the same amino acid have been described for short incubation periods, e. g. B. suis and B. melitensis are known to oxidize D-alanine more rapidly than the L-isomer [40] or B. abortus oxidized L-glutamic acid and L-asparagine rapidly whereas relatively slight activity was obtained with the D-isomers [38]. Differences in the metabolization rate could not be used for differentiation in our multi-substrate test. As many substrates were tested at the same time the incubation period was prolonged to 48 hours to ensure that each substrate was completely utilized. 
With a few exceptions, there are only minor differences in the general utilization of D- and L-isomers of amino acids within the same species [41]. Therefore both isomers of the same amino acid were only included three times in the Micronaut ${ }^{\text {tix }}$ plate, i.e. D-/L-proline, D-/Lalanine, and D-/L-serine. In our experiments, opposing metabolic activity could be observed for the different isomers of proline in B. abortus bv 3, B. suis bv 2, and $B$. canis, for the isomers of alanine in $B$. canis and $B$. neotomae, and for the isomers of serine in $B$. suis bv 1 , 2, and 4, B. ovis, B. microti and B. inopinata.

Further, substrate concentration may influence the metabolic activity of Brucella [34,38]. Although sample volumes are different in Taxa Profile ${ }^{\mathrm{Tm}}$ and Micronaut ${ }^{\mathrm{Tm}}$ plates the final substrate concentration is the same. Hence, apparently contradictory results in these two test systems which could be observed in our study cannot be explained by different concentrations of the same compound.

Because of the small volumes used in the Taxa Profile ${ }^{\text {tux }}$ plate turbidity could not be measured due to technical limitations. Therefore the indicator phenol red was added to colorimetrically measure respiration. In contrast, in the 96-well Micronaut ${ }^{\mathrm{Tm}}$ plate turbidity as a measure of bacterial growth was determined. The measurement of respiration instead of growth is much more sensitive since bacteria may respond metabolically by respiring but not by growing [42]. Hence, this effect may have led to differing results for the utilization of the same substrate on the two platforms. However, respiration could not be used in the genus Brucella since some strains are dependent on $\mathrm{CO}_{2}$ which catalyzes abiotic reduction of the dye.

As most metabolic pathways are encoded within the Brucella genome brucellae might present as fastidious due to slow growth. Although the genome sequence of $B$. microti is almost identical to that of $B$. suis with an overall sequence identity of $99.84 \%$ in aligned regions, phenotypically these species differ significantly which might be caused by variable gene regulations and different growth patterns [43].

Both respirometry and tetrazolium reduction assays proved that $B$. abortus is characteristically stimulated by L-alanine, L-asparagine and L-glutamate [30]. In contrast, the Micronaut ${ }^{\mathrm{tat}}$ results were heterogeneous for $\mathrm{L}$-alanine in B. abortus strains. The differences in metabolic activity observed between these methods might be caused by the cut-off selected in our experiments. Deduced from the OD values measured with the Micronaut ${ }^{\text {in }}$ system three levels of substrate utilization could be defined: no/weak metabolic activity (-), moderate metabolic activity $(+)$, and strong metabolic activity (++) [Additional file 7]. The different levels of oxidative metabolic activity on amino acid and carbohydrate substrates determined by Micronaut $^{\mathrm{ma}}$ agreed with the oxygen uptake levels for most substrates measured by conventional manometric techniques [25]. However, owing to the dispersion of the individual OD values, quantitative differences are of limited practical relevance. The selection of cut-offs which delineated positive and negative metabolic activity greatly contributed to the clarification of the presentation of substrate utilization. Of course, the limit between two activity patterns is rather artificial.

\section{Conclusions}

The results of the comprehensive biotyping study presented evidence that species of the genus Brucella can be correctly identified by their metabolic patterns. Although a range of metabolic properties allows clustering of Brucella into species and biovars clearly defined boundaries do not always exist.

Based on a selection of 93 different substrates out of 570 initially tested, a Brucella specific 96-well Micronaut $^{\text {tix }}$ microtiter plate was developed and successfully evaluated in a large panel of Brucella strains comprising all currently known species and biovars. Although the Micronaut $^{\text {tw }}$ system still requires a biological safety cabinet throughout the procedure it is much easier to handle and does not require the preparation of specific reagents leading to quicker results than conventional microbiological methods. Hence, the Micronaut ${ }^{\mathrm{m}} \mathrm{s}$ system may replace or at least complement time-consuming tube testing. Furthermore, an easy to handle identification software facilitates its applicability for routine use.

The newly developed Brucella specific 96-well Micronaut ${ }^{\mathrm{tm}}$ plate fulfilled the performance criteria recommended for a typing assay, i.e. typeability, reproducibility, stability and discriminatory power. Although we were not able to examine the epidemiological concordance of our biotyping results, there are definite indications that the metabolic profiles of different isolates match within the same outbreak [16]. The Micronaut ${ }^{\mathrm{tw}}$ system has also proven to be invaluable in the characterization of otherwise untypable new species. However, reference and new strains should always be tested in the same series because the differences in oxidative metabolic profiles may not only be qualitative but also quantitative.

Biodiversity of Brucella spp. also reflects taxonomic (natural and evolutionary) relationships that exist between and among the organisms sequestered and clustered within the classification scheme. Hence, the Micronaut ${ }^{\mathrm{tm}}$ system is not only a diagnostic assay it can be a striking tool in functional taxonomy of the genus Brucella.

Our results may raise the question if the widely accepted biotyping scheme based on only a few phenotypic features is sufficient to get a clear idea of the true composition of the genus Brucella and will meet future demands. The new diagnostic approach presented in this study may help to overcome these limitations. 


\section{Methods}

\section{Brucella strains}

Brucella spp. were characterized by classical microbiological methods according to Alton et al. (1988) [2]. Comprehensive biochemical phenotyping was performed on the Brucella reference strains representing all currently known species and their biovars as well as on up to 7 field isolates per species and biotype as far as available (Table 2). The consecutively established Brucella specific 96-well microtiter plate was evaluated testing the reference strains and a broad range of Brucella isolates (a total of 113 strains) originating from various animal hosts and human patients, i.e. B. melitensis bv $1(\mathrm{n}=8)$, bv $2(\mathrm{n}=14)$ and bv $3(\mathrm{n}=11) ; B$. abortus bv $1(\mathrm{n}=$ $9)$, bv $2(\mathrm{n}=2)$, bv $3(\mathrm{n}=5)$, bv $4(\mathrm{n}=6)$, bv $5(\mathrm{n}=$ $1)$, bv $6(\mathrm{n}=3)$, bv $7(\mathrm{n}=1)$ and bv $9(\mathrm{n}=3)$; B. suis bv $1(\mathrm{n}=6)$, bv $2(\mathrm{n}=8)$, bv $3(\mathrm{n}=1)$, bv $4(\mathrm{n}=2)$ and bv $5(\mathrm{n}=1)$; B. canis $(\mathrm{n}=5)$, B. ovis $(\mathrm{n}=4), B$. neotomae $(\mathrm{n}=1), B$. pinnipedialis $(\mathrm{n}=8)$ and $B$. ceti $(\mathrm{n}$ $=1)$, B. microti $(\mathrm{n}=10)$, B. inopinata $(\mathrm{n}=1)$, and two atypical strains according to the hitherto existing biotyping scheme (Table 2). Isolates of diverse geographical origin were deliberately selected to gain a large variety of strains.

Table 2 Brucella strains tested for metabolic activity

\begin{tabular}{|c|c|c|c|c|c|c|}
\hline \multirow[t]{2}{*}{ Species } & \multirow[t]{2}{*}{ Biovar } & \multirow[t]{2}{*}{ Strain } & \multirow{2}{*}{$\begin{array}{l}\text { Culture } \\
\text { collection }\end{array}$} & \multirow[t]{2}{*}{ Host } & \multicolumn{2}{|c|}{ Number of field isolates } \\
\hline & & & & & $\begin{array}{c}\text { Taxa Profile }^{\mathrm{TM}}(570 \\
\text { substrates) }\end{array}$ & $\begin{array}{c}\text { Micronaut }{ }^{\mathrm{TM}} \text { Brucella plate (93 } \\
\text { substrates) }\end{array}$ \\
\hline & 1 & 544 & NCTC 10093 & Cattle & 6 & 8 \\
\hline & 2 & $86 / 8 / 59$ & NCTC 10501 & Cattle & 1 & 1 \\
\hline & 3 & Tulya & NCTC 10502 & Human & 4 & 4 \\
\hline \multirow[t]{6}{*}{ B. abortus } & 4 & 292 & NCTC 10503 & Cattle & 5 & 5 \\
\hline & 5 & B3196 & NCTC 10504 & Cattle & 0 & 0 \\
\hline & 6 & 870 & NCTC 10505 & Cattle & 3 & 2 \\
\hline & $7^{*}$ & 63175 & NCTC 10506 & Cattle & 0 & 0 \\
\hline & 9 & C68 & NCTC 10507 & Cattle & 2 & 2 \\
\hline & 1 & $16 \mathrm{M}$ & NCTC 10094 & Goat & 4 & 7 \\
\hline \multirow{4}{*}{$\begin{array}{l}\text { B. } \\
\text { melitensis }\end{array}$} & 2 & $63 / 9$ & NCTC 10508 & Goat & 5 & 13 \\
\hline & 3 & Ether & NCTC 10509 & Goat & 4 & 10 \\
\hline & 1 & 1330 & NCTC 10316 & Swine & 4 & 5 \\
\hline & 2 & Thomsen & NCTC 10510 & Swine & 6 & 7 \\
\hline \multirow[t]{3}{*}{ B. suis } & 3 & 686 & NCTC 10511 & Swine & 1 & 0 \\
\hline & 4 & 40 & AFSSA ${ }^{b}$ Ref. 40 & Reindeer & 1 & 1 \\
\hline & 5 & 513 & AFSSA Ref. 513 & Wild rodent & 0 & 0 \\
\hline B. canis & & RM6/66 & NCTC 10854 & Dog & 4 & 4 \\
\hline B. ovis & & $63 / 290$ & NCTC 10512 & Sheep & 2 & 3 \\
\hline B. neotoma & & $5 K 33$ & NCTC 10084 & Desert rat & 0 & 0 \\
\hline \multicolumn{2}{|c|}{ B. pinnipedialis } & & NCTC 12890 & $\begin{array}{l}\text { Common } \\
\text { seal }\end{array}$ & 7 & 7 \\
\hline \multicolumn{3}{|l|}{ B. ceti } & NCTC 12891 & Porpoise & 0 & 0 \\
\hline \multicolumn{2}{|l|}{ B. microti } & & $\mathrm{CCM}^{\mathrm{C}} 4915$ & $\begin{array}{l}\text { Common } \\
\text { vole }\end{array}$ & 1 & 9 \\
\hline \multicolumn{2}{|l|}{ B. inopinata } & BO1 & $\mathrm{BCCN}^{\mathrm{d}}$ 09-01 & Human & 0 & 0 \\
\hline \multicolumn{2}{|l|}{ Unknown } & & $\mathrm{BfR}^{\mathrm{e}} 11.1 .001 / 002$ & Fox & 0 & 2 \\
\hline \multicolumn{2}{|l|}{ Total } & $\begin{array}{l}23 \text { reference } \\
\text { strains }\end{array}$ & & & 60 field isolates & 90 field isolates \\
\hline \multicolumn{7}{|c|}{$\begin{array}{l}\text { Brucella reference strains and overview of field isolates tested with the Taxa Profile }{ }^{\mathrm{TM}} \text { system and the newly developed Brucella specific Micronaut }{ }^{\mathrm{TM}} \text { microtiter } \\
\text { plate. }\end{array}$} \\
\hline \multicolumn{7}{|c|}{ a NCTC: National Collection of Type Cultures. } \\
\hline \multicolumn{7}{|c|}{ b AFSSA: Agence Française de Sécurité Sanitaire des Aliments. } \\
\hline \multicolumn{7}{|c|}{${ }^{\mathrm{c}} \mathrm{CCM}$ : Czech Collection of Microorganisms. } \\
\hline \multicolumn{7}{|c|}{${ }^{\mathrm{d}}$ BCCN: Brucella Culture Collection from Nouzilly. } \\
\hline \multicolumn{7}{|c|}{ e BfR: Bundesinstitut für Risikobewertung. } \\
\hline
\end{tabular}


Various strains initially tested with the 384-well Taxa Profile $^{\mathrm{TM}}$ plates were re-evaluated using the newly developed 96-well plate. In addition, a limited selection of closely related and clinically relevant bacteria was tested, i.e. Acinetobacter lwoffii (DSM 2403), Yersinia enterocolitica O:9 (IP-383 RKI/Paris), Ochrobactrum intermedium (CCUG 24964), O. anthropi (DSM 6882), Enterococcus faecalis (DSM 2570), Escherichia coli (DSM 1103), Pseudomonas aeruginosa (DSM 1117), and Staphylococcus aureus (DSM 2569).

\section{Culture and sample preparation}

All strains were grown on Brucella agar for $48 \mathrm{~h}$ at $37^{\circ} \mathrm{C}$ with or without $10 \% \mathrm{CO}_{2}$ depending on the needs of the particular species. Horse serum (10\%) was added to the culture medium to facilitate the growth of B. ovis. Colony material was harvested and solubilised in $0.1 \%$ buffered sodium chloride peptone (from potatoes) solution and in sterile $0.9 \% \mathrm{NaCl}$ for use in profile $\mathrm{A}$ or $\mathrm{C}$ plates and profile $\mathrm{E}$ plates, respectively. The turbidity of the bacterial suspension was adjusted to a 2.0 McFarland standard. Each well of the 384- and 96-well plates was inoculated with $25 \mu \mathrm{l}$ and $100 \mu \mathrm{l}$ of the respective preparation, respectively. The microtiter plates were incubated at $37^{\circ} \mathrm{C}$ for $48 \mathrm{~h}$ before reading.

\section{Brucella phenotyping}

The metabolic activity of Brucella was comprehensively assessed using the Taxa Profile ${ }^{\mathrm{TM}}$ system (Merlin Diagnostika, Bornheim-Hersel, Germany) based on 384-well microtiter plates coated with various substrates. The Taxa Profile ${ }^{\mathrm{TM}}$ A microtiter plate allows testing of 191 different amines, amides, amino acids, other organic acids and heterocyclic and aromatic substrates [Additional file 1]. The Taxa Profile ${ }^{\mathrm{Tm}} \mathrm{C}$ microtiter plate enables the analysis of 191 different mono-, di-, tri- and polysaccharides and sugar derivates [Additional file 2]. Using the Taxa Profile ${ }^{\mathrm{TM}}$ E microtiter plate another 188 substrates to determine enzymatic activity were tested: 95 amino peptidase- and protease-reactions, 76 glycosidase-, phosphatase- and other esterase-reactions, and 17 classic reactions [Additional file 3]. According to manufacturer's instructions supplementary reagents were added to some wells of the Taxa Profile ${ }^{\mathrm{TM}} \mathrm{E}$ microtiter plates to visualize substrate utilization. In addition, the indicator phenol red was added to all wells of the Taxa Profile $^{\mathrm{Tm}} \mathrm{A}$ and $\mathrm{C}$ microtiter plates to optimize detection. The blank value was measured for each biochemical reaction on the same plate and subtracted from measured values. In order to assess inter-assay variability five independent experiments per strain were conducted.

For evaluation of the newly developed Brucella specific 96-well microtiter plate three trials per strain were run independently. Intra-assay variability was assessed with the reference strains testing all substances twice within the same experiment. Since the blank values measured on extra plates proved to be constant a fixed mean value of each substrate was subtracted from the measured data.

\section{Data acquisition and analysis}

Turbidity and colour change were measured photometrically using a Multiskan Ascent ${ }^{\oplus}$ photometer (Labsystems, Helsinki, Finland) at a wave length of $405 \mathrm{~nm}, 540$ $\mathrm{nm}$ and $620 \mathrm{~nm}$ according to manufacturer's recommendations. Optimal OD cut-off values were empirically adapted from the preliminary test results of the 384wells Taxa Profile ${ }^{\mathrm{TM}}$ microtiter plates.

Stable and discriminatory markers were selected to design a 96-well Micronaut ${ }^{\mathrm{Tm}}$ plate (Figure 2) to identify bacteria of the genus Brucella and to classify their species and biovar.

Dendrograms were deduced from the biotyping data using SPSS version 12.0.2 (SPSS Inc., Chicago, IL, USA). First of all, three different character data sets were defined following the metabolic activity tested (Taxa Profile $^{\text {тм }}$ A ("amino acids"), C ("carbohydrates"), and E ("other enzymatic reactions")). Each character was considered as equal within the particular data set. Both the raw OD data and the binary coded data based on the empirically set cut-off were analyzed using the Pearson coefficient and the categorical coefficient, respectively. Hierarchical cluster analysis was performed by the Ward's linkage algorithm, and a dendrogram was generated. If necessary, analysis was repeated within each cluster for further discrimination. Secondly, a separate data analysis of the 23 Brucella reference strains representing the currently known species and biovars was performed including all biochemical reactions of the Taxa Profile ${ }^{\mathrm{Tm}}$ system or exclusively the substrates selected for the newly developed plate. Finally, the whole collective of 113 strains tested with the Brucella specific Micronaut ${ }^{\mathrm{TM}}$ microtiter plate was analyzed to prove the diagnostic system. An identification table presenting quantitative and qualitative metabolic activity was created [Additional file 7] and the specificity of the test system to differentiate Brucella species and biovars was calculated (Table 1).

\section{Additional material}

Additional file 1: List of biochemical reactions tested with the Taxa Profile $^{T M}$ A plate. The Taxa Profile ${ }^{T M}$ A microtiter plate allows testing of 191 different amines, amides, amino acids, other organic acids and heterocyclic and aromatic substrates.

Additional file 2: List of biochemical reactions tested with the Taxa Profile $^{T M} \mathrm{C}$ plate. The Taxa Profile ${ }^{\mathrm{TM}} \mathrm{C}$ microtiter plate enables the analysis of 191 different mono-, di-, tri- and polysaccharides and sugar derivates. 
Additional file 3: List of biochemical reactions tested with the Taxa Profile $^{\text {TM }} \mathrm{E}$ plate. The Taxa Profile ${ }^{T M} \mathrm{E}$ microtiter plate is configured to determine the enzymatic activity of 95 amino peptidases and proteases, 76 glycosidases, phosphatases and other esterases, and also includes 17 classic reactions.

Additional file 4: Cluster analysis of Brucella reference and field strains based on their amino acid metabolism. Cluster analysis of 83 Brucella and 2 Ochrobactrum strains based on 191 biochemical reactions tested with the Taxa Profile ${ }^{\mathrm{TM}}$ A plate. Hierarchical cluster analysis was performed by the Ward's linkage algorithm using the raw OD data.

Additional file 5: Cluster analysis of Brucella reference and field strains based on their carbohydrate metabolism. Cluster analysis of 83 Brucella and 2 Ochrobactrum strains based on 191 biochemical reactions tested with the Taxa Profile ${ }^{\mathrm{TM}} \mathrm{C}$ plate. Hierarchical cluster analysis was performed by the Ward's linkage algorithm using the raw OD data.

Additional file 6: Cluster analysis of Brucella reference and field strains based on specific enzymatic reactions. Cluster analysis of 83 Brucella and 2 Ochrobactrum strains based on 188 biochemical reactions tested with the Taxa Profile ${ }^{\text {TM }} E$ plate. Hierarchical cluster analysis was performed by the Ward's linkage algorithm using the raw OD data.

Additional file 7: Metabolic activity of Brucella strains. Relative frequency (\%) of positive and negative metabolic activity among 23 Brucella reference strains and 90 field isolates (Table 2) observed for the 93 substances tested in the Brucella specific Micronaut ${ }^{T M}$ plate. Both quality and relative quantity are presented: - no metabolic activity (highlighted in green), + moderate metabolic activity (in orange), ++ strong metabolic activity (in red).

Additional file 8: Separation of Brucella spp. from clinically relevant bacteria. Relative frequency (\%) of positive metabolic activity among Brucella and other bacteria observed for HP, Pyr- $\beta$ NA (Pyr), urease, and NTA. Four enzymatic reactions which were revealed by the MicronautIDS database screening clearly discriminated Brucella from clinically relevant bacteria of other genera.

\section{Acknowledgements}

The project was partially supported by research funds of the Bundeswehr Medical Service. We are grateful to Dr. Esther Pfeil (Merlin Diagnostika, Bornheim-Hersel, Germany) who compared our results with the MicronautIDS database of Merlin Diagnostika. We thank Dr. Erwin Hofer (Institute for Veterinary Disease Control, Mödling, Austria) for providing the fox isolates. Finally, we also thank our colleague Dr. Anne Mayer-Scholl for critical reading of the manuscript.

\section{Author details}

${ }^{1}$ Federal Institute for Risk Assessment, Diedersdorfer Weg 1, D-12277 Berlin, Germany. ${ }^{2}$ RWTH Aachen University, Department of Internal Medicine III, Pauwelsstraße 30, D-52074 Aachen, Germany. ${ }^{3}$ Bundeswehr Institute of Microbiology, Department of Bacteriology, Neuherbergstraße 11, D-80937 Munich, Germany. ${ }^{4}$ Friedrich Loeffler Institute, Institute of Bacterial Infections and Zoonoses, Naumburgerstraße 96a, D-07743 Jena, Germany.

\section{Authors' contributions}

$S A D, H N, H T, K N, B A$ and $A H$ were responsible for the study design. $P B, C G$, $\mathrm{KN}, \mathrm{SAD}$ and $\mathrm{HS}$ were in charge of strain collection, selection and the biotyping work. SAD, PB and WK performed cluster analysis and checked the dataset for errors. KN, PB, SAD and HN designed the Brucella specific Micronaut $^{\mathrm{TM}}$ microtiter plate. SAD wrote the report. KN, HN and WK helped to draft the manuscript. All authors read, commented and approved the final article.

Received: 28 June 2010 Accepted: 23 October 2010 Published: 23 October 2010

\section{References}

1. Al Dahouk S, Tomaso H, Nöckler K, Neubauer H, Frangoulidis D: Laboratorybased diagnosis of brucellosis - a review of the literature. Part I: techniques for direct detection and identification of Brucella spp. Clin Lab 2003, 49:487-505.

2. Alton GG, Jones LM, Angus RD, Verger JM: Techniques for the brucellosis laboratory Paris : Institut National de la Recherche Agronomique; 1988.

3. Morgan WJB, Corbel MJ: Recommendations for the description of species and biotypes of the genus Brucella. Develop Biol Standard 1976, 31:27-37.

4. Corbel MJ, Brinley-Morgan WJ: Genus Brucella. In Bergey's Manual of Systematic Bacteriology. Volume 1. Edited by: Krieg NR, Holt JG. Baltimore: Williams and Wilkins; 1984:370.

5. Foster G, Osterman BS, Godfroid J, Jacques I, Cloeckaert A: Brucella ceti sp. nov. and Brucella pinnipedialis sp. nov. for Brucella strains with cetaceans and seals as their preferred hosts. Int J Syst Evol Microbiol 2007, 57:2688-2693.

6. Scholz HC, Hubalek Z, Sedlacek I, Vergnaud G, Tomaso H, Al Dahouk S, Melzer F, Kämpfer $P$, Neubauer $H$, Cloeckaert A, Maquart M, Zygmunt MS, Whatmore A, Falsen E, Bahn P, Göllner C, Pfeffer M, Huber B, Busse HJ Nöckler K: Brucella microti sp. nov., isolated from the common vole Microtus arvalis. Int J Syst and Evol Microbiol 2008, 58:375-382.

7. Scholz HC, Hofer E, Vergnaud G, Le Flèche P, Whatmore A, Al Dahouk S, Pfeffer M, Krüger M, Cloeckaert A, Tomaso H: Isolation of Brucella microti from mandibular lymph nodes of red foxes, Vulpes vulpes, in Lower Austria. Vector Borne Zoonotic Dis 2009, 9:153-155.

8. Scholz HC, Hubalek $Z$, Nesvadbova J, Tomaso H, Vergnaud G, Le Flèche P, Whatmore AM, Al Dahouk S, Krüger M, Lodri C, Pfeffer M: Isolation of Brucella microti from soil. Emerg Infect Dis 2008, 14:1316-1317.

9. Scholz HC, Nöckler K, Göllner C, Bahn P, Vergnaud G, Tomaso H, Al Dahouk S, Kämpfer P, Cloeckaert A, Maquart M, Zygmunt MS, Whatmore AM, Pfeffer M, Huber B, Busse HJ, De BK: Brucella inopinata sp. nov, isolated from a breast implant infection. Int J Syst Evol Microbiol 2010, 60:801-808.

10. Banai M, Mayer I, Cohen A: Isolation, identification and characterization in Israel of Brucella melitensis biovar 1 atypical strains susceptible to dyes and penicillin, indicating the evolution of a new variant. J Clin Microbiol 1990, 28:1057-1059.

11. Ewalt DR, Forbes LB: Atypical isolates of Brucella abortus from Canada and the United States characterized as dye sensitive with $M$ antigen dominant. J Clin Microbiol 1987, 25:698-701.

12. Barham WB, Church P, Brown JE, Paparello S: Misidentification of Brucella species with use of rapid bacterial identification systems. Clin Infect Dis 1993, 17:1068-1069.

13. Elsaghir AA, James EA: Misidentification of Brucella melitensis as Ochrobactrum anthropi by API20NE. J Med Microbiol 2003, 52:441-442.

14. Meyer ME, Morgan WJB: Metabolic characterization of Brucella strains that show conflicting identity by biochemical and serological methods. Bull World Health Organ 1962, 26:823-827.

15. Cameron HS, Meyer ME: Metabolic studies on Brucella neotomae (Stoenner and Lackman). J Bacteriol 1958, 76:546-548.

16. Wundt W: Stoffwechseluntersuchungen als experimentelle Grundlage zur Einteilung des Genus Brucella. Zentralbl Bakteriol Orig 1963, 189:389-404.

17. Meyer ME: Metabolic characterization of the genus Brucella. III. Oxidative metabolism of strains that show anomalous characteristics by conventional determinative methods. J Bacteriol 1961, 82:401-410.

18. Meyer ME: Metabolic characterization of the genus Brucella. IV. Correlation of oxidative metabolic patterns and susceptibility to Brucella bacteriophage, type abortus, strain 3. J Bacteriol 1961, 82:950-953.

19. Meyer ME: Metabolic and bacteriophage identification of Brucella strains described as Brucella melitensis from cattle. Bull World Health Organ 1962, 26:829-831.

20. Jahans KL, Foster G, Broughton ES: The characterization of Brucella strains isolated from marine mammals. Vet Microbiol 1997, 57:373-382.

21. Jensen AE, Ewalt DR, Cheville NF, Thoen CO, Payeur JB: Determination of stability of Brucella abortus RB51 by use of genomic fingerprint, oxidative metabolism, and colonial morphology and differentiation of strain RB51 from B. abortus isolates from bison and elk. J Clin Microbiol 1996, 34:628-633.

22. Meyer ME: Inter- and intra-strain variants in the genus Brucella. Develop Biol Standard 1984, 56:73-83. 
23. Meyer ME, Cameron HS: Metabolic characterization of the genus Brucella. I. Statistical evaluation of the oxidative rates by which type I of each species can be identified. J Bacteriol 1961, 82:387-395.

24. Meyer ME, Cameron HS: Metabolic characterization of the genus Brucella. II. Oxidative metabolic patterns of the described biotypes. J Bacteriol 1961, 82:396-400.

25. Verger JM, Grayon M: Oxidative metabolic profiles of Brucella species. Ann Sclavo 1977, 19:45-60.

26. Maquart $M$, Le Flèche $P$, Foster $G$, Tryland $M$, Ramisse $F$, Diønne $B$, Al Dahouk S, Jacques I, Neubauer H, Walravens K, Godfroid J, Cloeckaert A, Vergnaud G: MLVA-16 typing of 295 marine mammal Brucella isolates from different animal and geographic origins identifies 5 major groups within Brucella ceti and Brucella pinnipedialis. BMC Microbiol 2009, 9:145.

27. Al Dahouk $S$, Le Flèche $P$, Nöckler $K$, Jacques I, Grayon M, Scholz HC, Tomaso H, Vergnaud G, Neubauer H: Evaluation of Brucella MLVA typing for human brucellosis. J Microbiol Methods 2007, 69:137-145.

28. Ewalt DR, Payeur JB, Martin BM, Cummins DR, Miller WG: Characteristics of a Brucella species from a bottlenose dolphin (Tursiops truncates). J Vet Diagn Invest 1994, 6:448-452.

29. Wong JD, Janda JM, Duffey PS: Preliminary studies on the use of carbon substrate utilization patterns for the identification of Brucella species. Diagn Microbiol Infect Dis 1992, 15:109-113.

30. Broughton ES, Jahans KL: The differentiation of Brucella species by substrate specific tetrazolium reduction. Vet Microbiol 1997, 51:253-271.

31. López-Merino A, Monnet DL, Hernández I, Sánchez NL, Boeufgras JM, Sandoval $H$, Freney J: Identification of Brucella abortus, B. canis, B. melitensis, and $B$. suis by carbon substrate assimilation tests. Vet Microbiol 2001, 80:359-363.

32. Cameron HS, Holm LW, Meyer ME: Comparative metabolic studies on the genus Brucella. I. Evidence of a urea cycle from glutamic acid metabolism. J Bacteriol 1952, 64:709-712.

33. Altenbern RA, Housewright RD: Carbohydrate oxidation and citric acid synthesis by smooth Brucella abortus, strain 19. Arch Biochem 1952, 36:345-356.

34. Gerhardt P, MacGregor DR, Marr AG, Olsen CB, Wilson JB: The metabolism of brucellae: the role of cellular permeability. J Bacteriol 1953, 65:581-586.

35. Meyer ME, Cameron HS: Species metabolic patterns within the genus Brucella. Am J Vet Res 1958, 19:754-758.

36. Al Dahouk S, Jubier-Maurin V, Scholz HC, Tomaso H, Karges W, Neubauer H, Köhler S: Quantitative analysis of the intramacrophagic proteome of the pathogen Brucella suis reveals metabolic adaptation to the late stage of cellular infection. Proteomics 2008, 8:3862-3870.

37. Al Dahouk S, Loisel-Meyer S, Scholz HC, Tomaso H, Kersten M, Harder A, Neubauer $\mathrm{H}$, Köhler $\mathrm{S}$, Jubier-Maurin V: Proteomic analysis of Brucella suis under oxygen deficiency reveals flexibility in adaptive expression of various pathways. Proteomics 2009, 9:3011-3021.

38. Gerhardt $P$, Levine $H B$, Wilson JB: The oxidative dissimilation of amino acids and related compounds by Brucella abortus. J Bacteriol 1950 60:459-467.

39. Essenberg RC, Seshadri R, Nelson K, Paulsen I: Sugar metabolism by brucellae. Vet Microbiol 2002, 90:249-261.

40. Cameron HS, Meyer ME: Comparative metabolic studies on the genus Brucella. II. Metabolism of amino acids that occur in the urea cycle. $J$ Bacteriol 1954, 67:34-37.

41. Sanders TH, Higuchi K, Brewer CR: Studies on the nutrition of Brucella melitensis. J Bacteriol 1953, 66:294-299.

42. Bochner BR: Global phenotypic characterization of bacteria. FEMS Microbiol Rev 2009, 33:191-205.

43. Audic S, Lescot M, Claverie JM, Scholz HC: Brucella microti: the genome sequence of an emerging pathogen. BMC Genomics 2009, 10:352.

44. Osterman B, Moriyón I: International Committee on Systematics of Prokaryotes, Subcommittee on the taxonomy of Brucella, Minutes of the meeting, 17 September 2003, Pamplona, Spain. Int I Syst Evol Microbiol 2006, 56:1173-1175.

doi:10.1186/1471-2180-10-269

Cite this article as: Al Dahouk et al:: Differential phenotyping of Brucella species using a newly developed semi-automated metabolic system.

BMC Microbiology 2010 10:269.

\section{Submit your next manuscript to BioMed Central and take full advantage of:}

- Convenient online submission

- Thorough peer review

- No space constraints or color figure charges

- Immediate publication on acceptance

- Inclusion in PubMed, CAS, Scopus and Google Scholar

- Research which is freely available for redistribution

Submit your manuscript at www.biomedcentral.com/submit
Ciomed Central 